\title{
The Difference in Prognostic Outcomes Between De Novo Stage IV and Recurrent Metastatic Patients with Hormone Receptor-positive, HER2-negative Breast Cancer
}

\author{
JUN YAMAMURA $^{1,2}$, SHUNJI KAMIGAKI ${ }^{1}$, JUNYA FUJITA $^{1}$, HIROKI OSATO $^{1}$ and YOSHIFUMI KOMOIKE ${ }^{2}$ \\ ${ }^{1}$ Department of Surgery, Sakai City Medical Center, Osaka, Japan; \\ ${ }^{2}$ Section of Breast and Endocrine Surgery, Department of Surgery, \\ Kindai University Faculty of Medicine, Sayama, Japan
}

\begin{abstract}
Background/Aim: Patients with de novo stage IV and recurrent metastatic breast cancer are often treated with the same strategies, although the difference in prognostic outcomes remains unclear. The objective of this retrospective chart review study was to compare the prognostic outcomes between two types of patients with hormone receptor-positive $(H R+)$, HER2-negative (HER2-) breast cancer. Patients and Methods: We estimated overall survival of the two groups and evaluated the progressive course of the disease using disease-free interval (DFI) and interval from the end of adjuvant treatment to the first recurrence (AFI). Results: We studied 172 patients with HR+/HER2- breast cancer, of which 65 were de novo and 107 were recurrent. Median OS between de novo and recurrent $B C$ was 4.85 and 3.45 years, respectively $(p=0.046)$. Recurrent patients with a $D F I<2$ years were found to have a significantly poorer prognosis than recurrent patients with a DFI $\geq 2$ years $(p=0.016)$ and de novo patients $(p=0.002)$. Similarly, recurrent patients with an $A F I<1$ year had a significantly poorer prognosis compared to de novo patients $(p=0.026)$. Conclusion: De novo patients had better prognoses than recurrent patients with $D F I<2$ years or $A F I<1$ year, likely due to their therapynaïve status or lower resistance to systemic treatment.
\end{abstract}

Breast cancer (BC) is the most common malignancy diagnosed in women, and leads to cancer mortality worldwide $(1,2)$. The

This article is freely accessible online.

Correspondence to: Jun Yamamura, Department of Surgery, Sakai City Medical Center, 1-1-1, Ebaraji-cho, Nishi-ku, Sakai City, Osaka, 593-8304, Japan, Tel: +81 722721199, Fax: +81 722729911, email: june10th68@gmail.com

Key Words: Breast cancer, de novo stage IV patients, recurrent, HER2-negative, HR-positive. majority of BC patients are diagnosed at an early disease stage, receive surgery and adjuvant treatment, and become long-term survivors who can be classified as disease-free (3-5). However, patients that develop metastatic disease during or after standard of care are generally incurable. In these cases a primary treatment goal is symptom palliation $(6,7)$. Patients with distant metastasis at initial diagnosis are classified as de novo stage IV metastatic patients and can be viewed as a distinct subgroup $(8,9)$, and these patients could be considered a poor prognostic group with incurable disease. However, a previous report suggested that de novo $\mathrm{BC}$ patients tend to have a better prognosis relative to recurrent $\mathrm{BC}$ patients (10). De novo $\mathrm{BC}$ patients may have a better response to systemic therapy due to their treatment-naïve status, while recurrent $\mathrm{BC}$ patients are more likely to show therapeutic resistance to systemic therapy after undergoing adjuvant treatment. There could also be biological differences other than the therapeutic factors between de novo and recurrent $\mathrm{BC}$ patients (11-13). Metastatic $\mathrm{BC}$ patients could form heterogeneous groups and present with varying metastatic patterns and prognostic outcomes, therefore requiring distinct treatment approaches based on BC subtype $(14,15)$. Treatment strategies have continuously advanced thanks to improved understanding of the heterogeneity of metastatic disease, which can have distant patterns of recurrence and varying prognostic impacts $(15,16)$. These approaches, which include endocrine therapies, targeted therapies, and various types of chemotherapies, are selected according to metastatic and prognostic patterns or BC subtypes based on immunohistochemical or molecular classifications (17). Although the difference in metastatic patterns and survival outcomes between de novo and recurrent $\mathrm{BC}$ patients is still unclear, these patients have often been treated with the same strategies. The objective of this retrospective study is to examine the prognostic outcomes between these groups of patients, both with the hormone receptor positive $(\mathrm{HR}+)$, and HER2-negative (HER2-) subtype. A better understanding of the metastatic course and prognostic factors for these patients 
Table I. Baseline characteristics at the initial diagnosis of distant metastasis.

\begin{tabular}{|c|c|c|c|}
\hline Characteristics & De novo BC & Recurrent BC & $p$-Value \\
\hline Number & $65(38 \%)$ & $107(62 \%)$ & \\
\hline Age & & & 0.983 \\
\hline$<50$ years & $12(18 \%)$ & $20(19 \%)$ & \\
\hline $50-70$ years & $16(25 \%)$ & $25(23 \%)$ & \\
\hline$\geq 70$ years & $37(57 \%)$ & $62(58 \%)$ & \\
\hline \multicolumn{4}{|c|}{ Stage (UICC Classification) } \\
\hline I & & $20(19 \%)$ & \\
\hline II & & $68(63 \%)$ & \\
\hline III & & $19(18 \%)$ & \\
\hline Histological Grade & & & 0.070 \\
\hline Low $(1+2)$ & $39(60 \%)$ & $74(69 \%)$ & \\
\hline High (3) & $19(29 \%)$ & $16(15 \%)$ & \\
\hline Unknown & $7(11 \%)$ & $17(16 \%)$ & \\
\hline \multicolumn{4}{|l|}{ First metastatic site } \\
\hline Multiple & $21(32 \%)$ & $45(42 \%)$ & 0.202 \\
\hline Visceral & $35(54 \%)$ & $57(53 \%)$ & 0.942 \\
\hline Liver & $12(18 \%)$ & $28(26 \%)$ & 0.246 \\
\hline Lung & $23(35 \%)$ & $26(24 \%)$ & 0.163 \\
\hline Bone & $33(51 \%)$ & $55(51 \%)$ & 0.936 \\
\hline Soft tissue & $23(35 \%)$ & $44(41 \%)$ & 0.454 \\
\hline Brain & 0 & $3(3 \%)$ & 0.173 \\
\hline \multicolumn{4}{|l|}{ Primary treatment } \\
\hline Chemotherapy & $28(43 \%)$ & $37(35 \%)$ & 0.265 \\
\hline Endocrine therapy & $37(57 \%)$ & $70(65 \%)$ & \\
\hline \multicolumn{4}{|l|}{ DFI } \\
\hline$<2$ years & & $20(19 \%)$ & \\
\hline $2-5$ years & & $47(44 \%)$ & \\
\hline$\geq 5$ years & & $40(37 \%)$ & \\
\hline \multicolumn{4}{|l|}{ AFI } \\
\hline$<1$ year & & $70(68 \%)$ & \\
\hline $1-3$ years & & $19(18 \%)$ & \\
\hline$\geq 3$ years & & $14(14 \%)$ & \\
\hline
\end{tabular}

BC: Breast cancer; DFI: disease-free interval; AFI: interval from the end of adjuvant treatment to the first recurrence.

could improve treatment decisions and provide clinical benefit. This analysis may also help in comprehending and interpreting current clinical research and indicate targets for future clinical studies of metastatic HR+/HER2- BC.

\section{Patients and Methods}

Patient characteristics. We retrospectively reviewed the clinical records of de novo and recurrent metastatic HR+/HER2- BC patients treated between January 2000 and March 2013 in Sakai City Medical Center, Japan. Cases of de novo metastatic BC were classified as stage IV by the UICC TNM classification of malignant tumors, seventh edition. Recurrent patients demonstrated a distant metastasis after removal of the primary breast cancer and standard adjuvant treatment, and patients with locoregional recurrence were excluded from this analysis. This study was approved by the institutional review board and all enrolled patients provided informed consent.
Table II. Median survival time for de novo and recurrent BC according to function of clinical characteristics.

\begin{tabular}{|c|c|c|}
\hline Characteristics & $\begin{array}{c}\text { De novo } \mathrm{BC} \\
\text { Median (range) }\end{array}$ & $\begin{array}{c}\text { Recurrent BC } \\
\text { Median (range) }\end{array}$ \\
\hline All & $2.91(0.11-11.76)$ & $2.28(0.02-10.55)$ \\
\hline \multicolumn{3}{|l|}{ Age at enrollment } \\
\hline$<50$ years & $3.71(0.39-10.57)$ & $2.63(0.02-5.47)$ \\
\hline $50-70$ years & $3.71(0.35-11.76)$ & $2.41(0.13-10.55)$ \\
\hline$\geq 70$ years & $0.97(0.11-5.13)$ & $1.92(0.10-6.56)$ \\
\hline \multicolumn{3}{|c|}{ Stage (UICC Classification) } \\
\hline I & & $3.13(0.19-6.63)$ \\
\hline II & & $2.41(0.02-10.55)$ \\
\hline III & & $1.78(0.25-6.33)$ \\
\hline \multicolumn{3}{|l|}{ Histological Grade } \\
\hline Low $(1+2)$ & $3.39(0.14-11.58)$ & $2.49(0.02-10.55)$ \\
\hline High (3) & $1.45(0.11-5.01)$ & $1.78(0.36-3.98)$ \\
\hline \multicolumn{3}{|l|}{ First metastatic site } \\
\hline Multiple & $1.46(0.26-11.76)$ & $2.28(0.19-10.55)$ \\
\hline Visceral & $2.09(0.14-11.76)$ & $2.39(0.13-10.55)$ \\
\hline Liver & $1.77(0.26-11.58)$ & $2.06(0.13-6.84)$ \\
\hline Lung & $1.84(0.14-11.76)$ & $2.02(0.19-10.55)$ \\
\hline Bone & $3.67(0.11-11.76)$ & $2.28(0.02-10.55)$ \\
\hline Soft tissue & $2.03(0.26-6.25)$ & $2.23(0.10-10.55)$ \\
\hline \multicolumn{3}{|l|}{ Primary treatment } \\
\hline Chemotherapy & $2.68(0.26-11.76)$ & $2.19(0.02-8.72)$ \\
\hline Endocrine therapy & $3.00(0.11-11.58)$ & $2.40(0.10-10.55)$ \\
\hline \multicolumn{3}{|l|}{ DFI } \\
\hline$<2$ years & & $2.03(0.02-6.26)$ \\
\hline $2-5$ years & & $2.50(0.10-10.55)$ \\
\hline$\geq 5$ years & & $2.15(0.19-8.72)$ \\
\hline \multicolumn{3}{|l|}{ AFI } \\
\hline$<1$ year & & $2.11(0.02-10.55)$ \\
\hline $1-3$ years & & $3.68(0.19-8.72)$ \\
\hline$\geq 3$ years & & $2.33(0.48-6.56)$ \\
\hline
\end{tabular}

BC: Breast cancer; DFI: disease-free interval; AFI: interval from the end of adjuvant treatment to the first recurrence.
Subtype classification. Estrogen receptor (ER) and progesterone receptor (PR) positivity was defined as score $>2$ by the Allread scoring system, and HR positivity was defined as ER and/or PR positive breast cancer. HER2 negativity was defined as an immunohistochemistry score of $0,1+$, or $2+$ with a negative fluorescence in situ hybridization result (a ratio <2.0).

Survival outcomes and progressive courses. The primary aim of this study was to assess differences in the survival outcomes and progressive courses of recurrent $\mathrm{BC}$ patients compared to de novo $\mathrm{BC}$ patients. Overall survival (OS) was defined as the time from the date of initial diagnosis to the time of death or last follow-up for de novo patients. For recurrent $\mathrm{BC}$ patients, OS was defined as the time from the date of the first distant metastasis to the time of death or last followup, and disease-free interval (DFI) was defined as the time from the diagnosis of primary nonmetastatic breast cancer to the date of the first distant metastasis. In this study, we also calculated the interval from the end of adjuvant treatment to the first distant metastasis (AFI). 


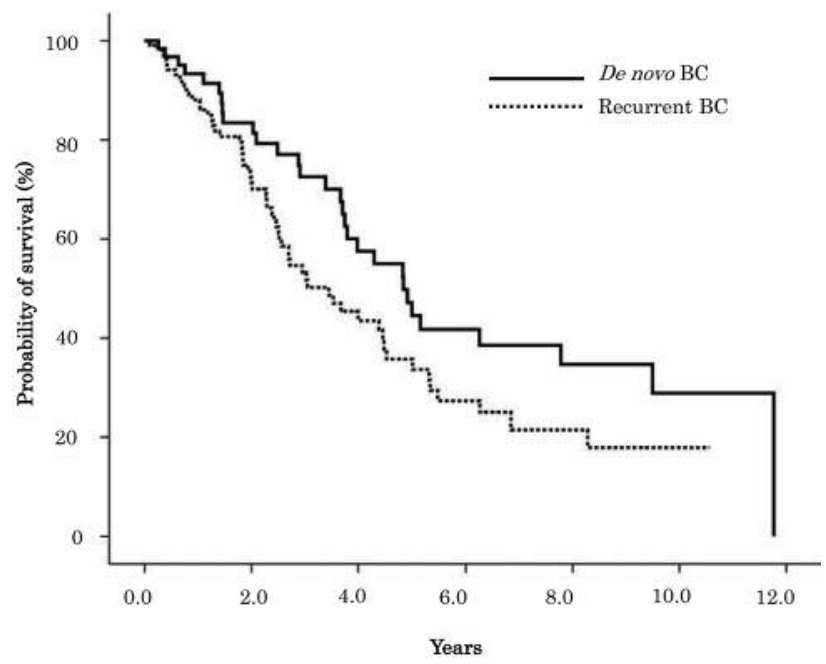

Figure 1. Overall survival for de novo and recurrent BC patients.

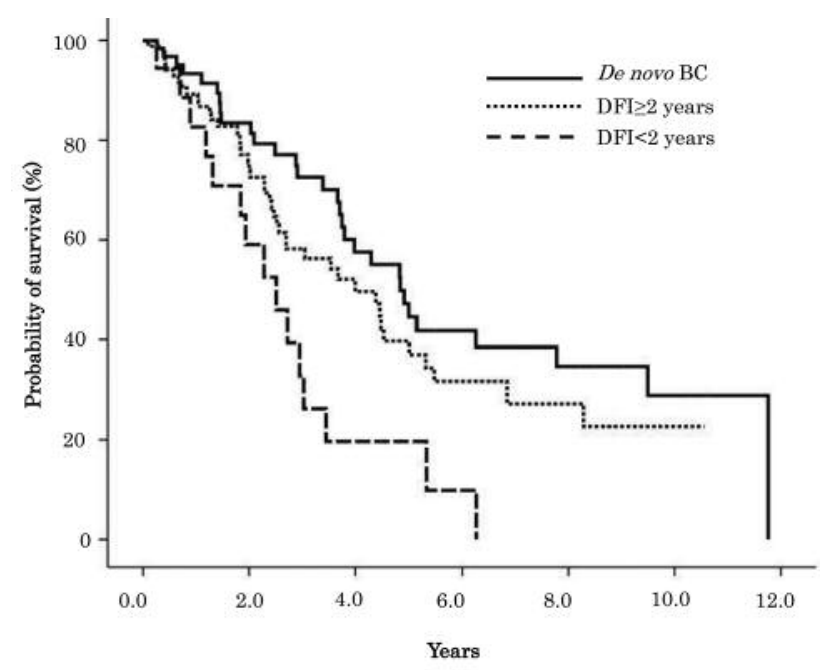

Figure 2. Overall survival for de novo and recurrent BC patients with $D F I \geq 2$ years and $D F I<2$ years.

Statistical analysis. OS plots were calculated using the KaplanMeier method, and the distributions of the survival curves were compared using log-rank tests. The Cox proportional hazard regression model was used to examine the prognostic evaluation between the groups using several prognostic indicators, such as patient and disease related clinicopathological factors, DFI, and AFI. A 95\% confidence interval (CI) was calculated for all hazard ratios (HR) in the Cox regression analysis. We evaluated the results of the univariate and multivariate Cox proportional hazards model with hazards ratios $(\mathrm{HR})>1.0$ indicating an increased risk of death. Statistical analyses was performed using the Statistical Software package SPSS (v.17.0; Chicago, IL, USA).

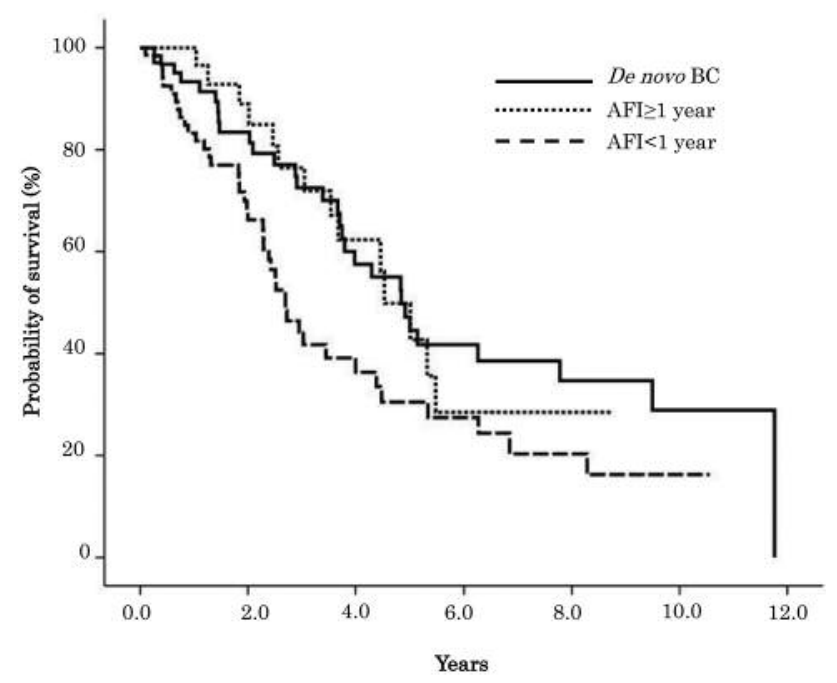

Figure 3. Overall survival for de novo and recurrent BC patients with $A F I \geq 1$ year and $A F I<1$ year.

\section{Results}

A total of 172 patients with HR+ and HER2- breast cancer were included in the analysis. Of these, 65 (38\%) were de novo and 107 (62\%) were recurrent metastatic patients. The characteristics of the patients and tumors at the initial disgnosis of distant metastasis were compared between the groups with a chi-square test and are summarized in Table I. In general, characteristics were well balanced between the groups. A majority of recurrent BC patients (68\%) developed initial distant metastases during or within one year after adjuvant treatment.

OS estimates according to clinical characteristics are summarized in Table II. Median OS between de novo and recurrent $\mathrm{BC}$ was 4.85 and 3.45 years, respectively, and the difference was statistically significant $(p=0.046)$ (Figure 1). Kaplan-Meyer survival curves show OS of de novo patients, recurrent $\mathrm{BC}$ patients with a $\mathrm{DFI}<2$ years, and reccurent $\mathrm{BC}$ patients with a $\mathrm{DFI} \geq 2$ years (Figure 2), as well as among de novo, recurrent $\mathrm{BC}$ patients with an $\mathrm{AFI}<1$ year, and recuurent patients with an $\mathrm{AFI} \geq 1$ year (Figure 3 ).

Tables III and IV respectively show the results of the univariate and multivariate analyses, performed to identify factors that affect the prognosis of de novo and recurrent $\mathrm{BC}$ patients. Univariate analysis showed that de novo $\mathrm{BC}$ patients had significantly better OS compared to recurrent BC patients ( $\mathrm{HR}=0.634,95 \% \mathrm{CI}=0.404-0.995, p=0.047)$. Recurrent $\mathrm{BC}$ patients with a $\mathrm{DFI}<2$ years were associated with a statistically significant increased risk of death compared to recurrent $\mathrm{BC}$ patients with a $\mathrm{DFI} \geq 2$ years $(\mathrm{HR}=2.046,95 \% \mathrm{CI}=1.130-3.706$, 
Table III. Univariate Cox regression analyses for overall survival.

\begin{tabular}{|c|c|c|c|}
\hline Variable & HR & $95 \% \mathrm{CI}$ & $p$-Value \\
\hline Age $(\geq 50$ years $v s .<50$ years $)$ & 1.411 & $0.861-2.311$ & 0.172 \\
\hline Histological grade (High (3) vs. Low $(1+2))$ & 1.806 & $0.997-3.269$ & 0.051 \\
\hline Multiple metastasis as initial metastatic site & 1.560 & $1.015-2.398$ & 0.043 \\
\hline Visceral metastasis as initial metastatic site & 1.151 & $0.754-1.757$ & 0.514 \\
\hline Lung metastasis as initial metastatic site & 1.422 & $0.846-2.390$ & 0.184 \\
\hline Liver metastasis as initial metastatic site & 2.725 & $1.751-4.255$ & $<0.001$ \\
\hline Bone metastasis as initial metastatic site & 0.855 & $0.560-1.304$ & 0.466 \\
\hline Soft tissue metastasis as initial metastatic site & 0.625 & $0.403-0.970$ & 0.036 \\
\hline De novo vs. Recurrent BC & 0.634 & $0.404-0.995$ & 0.047 \\
\hline $\mathrm{DFI}<2$ years $v s . \mathrm{DFI} \geq 2$ years & 2.046 & $1.130-3.706$ & 0.018 \\
\hline $\mathrm{DFI}<2$ years $v$ s. de novo $\mathrm{BC}$ & 2.929 & $1.539-5.574$ & 0.001 \\
\hline $\mathrm{DFI} \geq 2$ years $v$ s. de novo $\mathrm{BC}$ & 1.373 & $0.854-2.207$ & 0.191 \\
\hline $\mathrm{AFI}<1$ year $v s . \mathrm{AFI} \geq 1$ year & 1.783 & $0.968-3.287$ & 0.064 \\
\hline $\mathrm{AFI}<1$ year vs. de novo $\mathrm{BC}$ & 1.792 & $1.108-2.898$ & 0.017 \\
\hline $\mathrm{AFI} \geq 1$ year vs. de novo $\mathrm{BC}$ & 1.070 & $0.561-2.042$ & 0.837 \\
\hline
\end{tabular}

HR: Hazard ratio; CI: confidence interval; BC: breast cancer; DFI: disease-free interval; AFI: interval from the end of adjuvant treatment to the first recurrence.

Table IV. Multivariate Cox regression analyses for overall survival.

\begin{tabular}{llrr}
\hline Variable & HR & 95\%CI & $p$-Value \\
\hline De novo $v s$. Recurrent BC & 0.679 & $0.429-1.075$ & 0.099 \\
Multiple metastasis as initial metastatic site & 1.190 & $0.685-2.066$ & 0.538 \\
Liver metastasis as initial metastatic site & 2.849 & $1.745-4.651$ & $<0.001$ \\
Soft tissue metastasis as initial metastatic site & 0.604 & $0.359-1.015$ & 0.057 \\
\hline
\end{tabular}

HR: Hazard ratio; CI: confidence interval; BC: breast cancer.

$p=0.018)$, and de novo $\mathrm{BC}$ patients $(\mathrm{HR}=2.929,95 \% \mathrm{CI}=1.539$ $5.574, p=0.001)$. Recurrent $\mathrm{BC}$ patients with an $\mathrm{AFI}<1$ year were associated with a statistically significant increased risk of death compared to de novo $\mathrm{BC}$ patients $(\mathrm{HR}=1.792$, $95 \% \mathrm{CI}=1.108-2.898, p=0.017)$. There was no significant difference in risk of death among de novo $\mathrm{BC}$, recurrent $\mathrm{BC}$ with a $\mathrm{DFI} \geq 2$ years, and $\mathrm{AFI} \geq 1$ year patients. Multiple metastasis and liver metastasis as the first metastatic site both had an increased risk of death that was statistically significant. Liver metastasis as the first metastatic site was also associated with statistically significant increased risk of death in multivariate analysis.

\section{Discussion}

The increasing application of novel cancer treatments including chemotherapy, endocrine therapy, and molecular-targeted therapy has improved the survival of metastatic BC patients (18-20). These treament advances have resulted in decreasing recurrence rates for early-stage breast cancer (3-5). However, although the difference in the prognostic outcomes of metastatic diseases between de novo and recurrent $\mathrm{BC}$ patients is still unclear, these patients have often been treated using the same strategy. Previous reports have indicated that de novo $\mathrm{BC}$ patients have better survival than recurrent BC patients (1012), and our study also confirms these results. Metastatic BC forms heterogeneous groups with varying prognostic outcomes, requiring the development of a distinct treatment approach for each BC subtype (14-16). Most previous studies have reported survival outcomes of metastatic disease regardless of $\mathrm{BC}$ subtype in a large patient cohort and have not examined survival as a function of HR/HER2 status (10-12). Yardley et al. studied the survival outcomes of de novo and recurrent metastatic patients with HER2+ BC. The HER2+ BC subtype is distinct from other subtypes, and requires a specific treatment strategy, such as anti-HER2 targeted therapy (13). Our study is the first report of a survival analysis between $d e$ novo and recurrent $\mathrm{BC}$ patients with $\mathrm{HR}+/ \mathrm{HER} 2-\mathrm{BC}$. This is the most common BC subtype, and has a specific course of progression $(21,22)$. This subtype requires endocrine therapy but in some cases not chemotherapy in an adjuvant setting, and 
requires various treatment strategies in a metastatic setting (23, 24). A survival analysis of metastatic disease according to BC subtype could help in choosing an optimal treatment strategy in the patient population (25).

A majority of the recurrent BC patients in this study (68\%) developed metastatic disease during or within a year after adjuvant endocrine treatment, and may be likely to develop a resistance to systemic therapy. Therefore, recurrent BC patients with an $\mathrm{AFI}<1$ year had a poorer prognosis than patients with an $\mathrm{AFI} \geq 1$ year or de novo $\mathrm{BC}$ patients. Recurrent $\mathrm{BC}$ patients with a longer DFI or an $\mathrm{AFI} \geq 1$ year were also found to have a better prognosis, equivalent to de novo $\mathrm{BC}$ patients. There could be multiple reasons why recurrent $\mathrm{BC}$ patients with an $\mathrm{AFI}<1$ year or a shorter DFI have a poorer prognosis. One reason may be due to ineffective adjuvant treatment or poor carry-over effect of adjuvant treatment. BC patients who experienced a distant recurrence within or soon after adjuvant treatment may present with therapeutic resistance, also leading to poorer prognostic outcomes. In contrast, de novo disease is therapy-naive, and therefore likely to show less resistance to systemic therapy (10-12). BC patients with longer DFI or patients who developed a distant recurrence some years after adjuvant treatment may have a better response to systemic therapy. This study could contribute to a better understanding of differences in survival outcomes between de novo and recurrent HR+/HER2- BC, and even more conclusice results could be obtained with a larger spectrum of metastatic patients and treatment analysis.

In conclusion, we studied differences in survival outcomes between de novo and recurrent HR+/HER2- BC patients. De novo $\mathrm{BC}$ led to a better prognosis than recurrent $\mathrm{BC}$ with a $\mathrm{DFI}<2$ years or an AFI $<1$ year. This may be because of resistance to systemic therapy due to ineffective adjuvant treatment or poor carry-over effect. This study could aid in understanding valuable clinical relationships between these patients, leading to effective treatment strategies for HR+/HER2 - metastatic disease, as well as improved interpretation of results from clinical trials.

\section{Conflicts of Interest}

The Authors declare no conflicts of interest.

\section{References}

1 National Cancer Institute. Surveillance, Epidemiology, and End Results Program. SEER Stat Fact Sheets: Breast Cancer. http://seer.cancer.gov/statfacts/html/breast.html (accessed November 20, 2017).

2 Hortobagyi GN, de la Garza Salazar J, Pritchard K, Amadori D, Haidinger R, Hudis CA, Khaled H, Liu MC, Martin M, Namer M, O'Shaughnessy JA, Shen ZZ and Albain KS; ABREAST Investigators: The global breast cancer burden: variations in epidemiology and survival. Clin Breast Cancer 6: 391-401, 2005.
3 EBCTCG (Early Breast Cancer Trialists' Collaborative Group), McGale P, Taylor C, Correa C, Cutter D, Duane F, Ewertz M, Gray R, Mannu G, Peto R, Whelan T, Wang Y, Wang Z and Darby $S$ : Effect of radiotherapy after mastectomy and axillary surgery on 10-year recurrence and 20-year breast cancer mortality: meta-analysis of individual patient data for 8135 women in 22 randomised trials. Lancet 383: 2127-2135, 2014.

4 Early Breast Cancer Trialists' Collaborative Group (EBCTCG), Peto R, Davies C, Godwin J, Gray R, Pan HC, Clarke M, Cutter D, Darby S, McGale P, Taylor C, Wang YC, Bergh J, Di Leo A, Albain K, Swain S, Piccart M and Pritchard K: Comparisons between different polychemotherapy regimens for early breast cancer: meta-analyses of long-term outcome among 100,000 women in 123 randomised trials. Lancet 379: 432-444, 2012.

5 Early Breast Cancer Trialists' Collaborative Group (EBCTCG), Darby S, McGale P, Correa C, Taylor C, Arriagada R, Clarke M, Cutter D, Davies C, Ewertz M, Godwin J, Gray R, Pierce L, Whelan T, Wang Y and Peto R: Effect of radiotherapy after breastconserving surgery on 10-year recurrence and 15-year breast cancer death: meta-analysis of individual patient data for 10,801 women in 17 randomised trials. Lancet 378: 1707-1716, 2011.

6 Giuliani $\mathrm{J}$ and Bonetti A: Trends in survival for patients with metastatic breast cancer: is survival improving? Tumori 101: 347-352, 2015.

7 Chung CT and Carlson RW: Goals and objectives in the management of metastatic breast cancer. Oncologist 8: 514-520, 2003.

8 Mariotto AB, Etzioni R, Hurlbert M, Penberthy L and Mayer M: estimation of the number of women living with metastatic breast cancer in the United States. Cancer Epidemiol Biomarkers Prev 26: 809-815, 2017.

9 Bertaut A, Mounier M, Desmoulins I, Guiu S, Beltjens F, DarutJouve A, Ponnelle T, Arnould L and Arveux P: Stage IV breast cancer: a population-based study about prognostic factors according to HER2 and HR status. Eur J Cancer Care (Engl) 24: 920-928, 2015.

10 Dawood S, Broglio K, Ensor J, Hortobagyi GN and Giordano SH: Survival differences among women with de novo stage IV and relapsed breast cancer. Ann Oncol 21: 2169-2174, 2010.

11 den Brok WD, Speers CH, Gondara L, Baxter E, Tyldesley SK and Lohrisch CA: Survival with metastatic breast cancer based on initial presentation, de novo versus relapsed. Breast Cancer Res Treat 161: 549-556, 2017.

12 Lobbezoo DJ, van Kampen RJ, Voogd AC, Dercksen MW, van den Berkmortel F, Smilde TJ, van de Wouw AJ, Peters FP, van Riel JM, Peters NA, de Boer M, Peer PG and Tjan-Heijnen VC: Prognosis of metastatic breast cancer: are there differences between patients with de novo and recurrent metastatic breast cancer? Br J Cancer 112: 1445-1451, 2015.

13 Yardley DA, Kaufman PA, Brufsky A, Yood MU, Rugo H, Mayer M, Quah C and Yoo B, Tripathy D: Treatment patterns and clinical outcomes for patients with de novo versus recurrent HER2-positive metastatic breast cancer. Breast Cancer Res Treat 145: 725-734, 2014.

14 Soni A, Ren Z, Hameed O, Chanda D, Morgan CJ, Siegal GP and Wei S: Breast cancer subtypes predispose the site of distant metastases. Am J Clin Pathol 143: 471-478, 2015.

15 Kennecke H, Yerushalmi R, Woods R, Cheang MC, Voduc D, Speers CH, Nielsen TO and Gelmon K: Metastatic behavior of breast cancer subtypes. J Clin Oncol 28: 3271-3277, 2010. 
16 Lobbezoo DJ, van Kampen RJ, Voogd AC, Dercksen MW, van den Berkmortel F, Smilde TJ, van de Wouw AJ, Peters FP, van Riel JM, Peters NA, de Boer M, Borm GF and Tjan-Heijnen VC: Prognosis of metastatic breast cancer subtypes: the hormone receptor/HER2-positive subtype is associated with the most favorable outcome. Breast Cancer Res Treat 141: 507-514, 2013.

17 Kao KJ, Chang KM, Hsu HC and Huang AT: Correlation of microarray-based breast cancer molecular subtypes and clinical outcomes: implications for treatment optimization. BMC Cancer 11: 143, 2011

18 Wilson FR, Varu A, Mitra D, Cameron C and Iyer S: Systematic review and network meta-analysis comparing palbociclib with chemotherapy agents for the treatment of postmenopausal women with HR-positive and HER2-negative advanced/ metastatic breast cancer. Breast Cancer Res Treat 166: 167-177, 2017.

19 de Glas NA, Bastiaannet E, de Craen AJ, van de Velde CJ, Siesling S, Liefers GJ and Portielje JE: Survival of older patients with metastasised breast cancer lags behind despite evolving treatment strategies - a population-based study. Eur J Cancer 51: 310-316, 2015.

20 Andre F, Slimane K, Bachelot T, Dunant A, Namer M, Barrelier A, Kabbaj O, Spano JP, Marsiglia H, Rouzier R, Delaloge S and Spielmann M: Breast cancer with synchronous metastases: trends in survival during a 14 -year period. J Clin Oncol 22: 3302-3308, 2004.
21 Nakano M, Fujisue M, Tashima R, Okumura Y, Nishiyama Y, Ohsako T, Toyozumi Y, Arima N and Nishimura R: Survival time according to the year of recurrence and subtype in recurrent breast cancer. Breast 24: 588-593, 2015.

22 Howlader N, Altekruse SF, Li CI, Chen VW, Clarke CA, Ries LA and Cronin KA: US incidence of breast cancer subtypes defined by joint hormone receptor and HER2 status. J Natl Cancer Inst 106 (5), 2014.

23 Curigliano $\mathrm{G}$ and Criscitiello C: Maximizing the clinical benefit of anthracyclines in addition to taxanes in the adjuvant treatment of early breast cancer. J Clin Oncol 35: 2600-2603, 2017.

24 Barrios CH, Sampaio C, Vinholes J and Caponero R: What is the role of chemotherapy in estrogen receptor-positive, advanced breast cancer? Ann Oncol 20: 1157-1162, 2009.

25 Cossetti RJ, Tyldesley SK, Speers CH, Zheng Y and Gelmon KA: Comparison of breast cancer recurrence and outcome patterns between patients treated from 1986 to 1992 and from 2004 to 2008. J Clin Oncol 33: 65-73, 2015.

Received November 27, 2017

Revised December 16, 2017

Accepted December 18, 2017 